\title{
Routine neonatal examination: effectiveness of trainee paediatrician compared with advanced neonatal nurse practitioner
}

\author{
T W R Lee, R E Skelton, C Skene
}

\begin{abstract}
Objective-To compare the effectiveness of routine neonatal examination performed by senior house officers (SHOs) and advanced neonatal nurse practitioners (ANNPs).

Design-A prospective study of all infants referred to specialist orthopaedic, ophthalmology, and cardiology clinics. A standardised proforma was used to record details of the professional performing the neonatal check, any abnormalities discovered, source of ultimate referral to the specialist clinic, and specialist findings.

Results-527 eligible infants were recruited. For hip abnormalities, ANNPs displayed greater sensitivity than SHOs $(96 \% v 74 \% ; p<0.05)$. Similarly for eye abnormalities, ANNPs were more sensitive $(100 \% v 33 \% ; p<0.05)$. There were no significant differences between ANNPs and SHOs in terms of positive predictive values or effectiveness of detecting cardiac abnormalities. There was no difference in underlying incidence of abnormalities between the two hospitals.

Conclusion-ANNPs are significantly more effective in detecting abnormalities during the neonatal check. This has implications both for future workforce planning and current methods of medical training. (Arch Dis Child Fetal Neonatal Ed 2001;85:F100-F104)
\end{abstract}

Keywords: examination; advanced neonatal nurse practitioner; training

The role of the neonatal check remains controversial. ${ }^{1}$ The aim is to detect potentially serious conditions in apparently healthy neonates before they leave hospital, and to provide reassurance to the parents when the examination is normal. However, some conditions are not detectable in the immediate neonatal period. For instance, routine neonatal examination fails to detect more than half of babies with congenital heart disease. ${ }^{23}$ Only about $35 \%$ of congenital and infantile cataracts are picked up by the neonatal examination, ${ }^{4}$ yet the outcome after cataract surgery is optimised if operative intervention occurs before 6 weeks of age. ${ }^{5}$ Clinical screening for developmental dysplasia of the hip also fails to pick up a proportion of cases. $^{67}$ Many argue that the check could effectively be performed by a nurse practitioner or midwife. ${ }^{8}$ This would have the advantage of increased continuity of care, rather than introducing yet another professional to the new mother, as well as releasing paediatric trainees from a major service burden which has limited educational value.

Advanced neonatal nurse practitioners (ANNPs) are a relatively recent introduction to the field of neonatal care. As part of their training, they have formalised instruction in how to perform a neonatal check, ${ }^{910}$ together with clear teaching on the significant pathological variables they are looking for. ${ }^{11}$ In this regard, their training is probably more structured than many paediatric senior house officers (SHOs), who often have little formal training in how to perform an adequate neonatal examination. ${ }^{12}$ Currently most neonatal checks in the United Kingdom are performed by paediatric SHOs, but increasingly units are turning to ANNPs.

We have found no previous studies that compare the effectiveness of ANNP neonatal checks with those performed by paediatric SHOs, and it is thus the aim of this study to make this comparison.

\section{Methods}

A prospective study was conducted from 1 April 1999 to 31 March 2000 of all infants referred to specialist orthopaedic, ophthalmology, and cardiology clinics in Kingston upon Hull. A standardised proforma was used to record details of where the infant had been born, what sort of neonatal check had been carried out, and who had first discovered the abnormality that had led to the specialist referral.

There are two obstetric hospitals in the locality: Hull Maternity Hospital had 2783 live births during the study. Neonatal checks are carried out by paediatric SHOs. Castle Hill Hospital had 2244 live births and is staffed predominantly by ANNPs who perform the neonatal checks. There are no resident paediatricians at this hospital.

All children from Hull and East Yorkshire with a suspected diagnosis of, or significant risk factors for, developmental dysplasia of the hip are referred to the specialist orthopaedic paediatric hip clinic at Hull Royal Infirmary. Risk factors include breech presentation, family history, and structural talipes. Referral is also made if there is any clinical evidence of dislocatable or dislocated hip.

At the hip clinic, clinical examination and ultrasound are performed to determine whether the hip is normal, dysplastic requiring active intervention, or borderline requiring further observation (Graf grade IIa). ${ }^{13}$

Infants with suspected cataracts or other causes of impaired red reflex are referred to the ophthalmologists at Hull Royal Infirmary, 
Table 1 Summary of sources and findings of specialist orthopaedic hip referrals

\begin{tabular}{llll}
\hline & Normal hip & Abnormal hip & Total referred \\
\hline Paediatric SHOs & 156 & 20 & \\
$\quad$ Referred from neonatal check & 28 & 7 & 176 \\
$\quad$ Referred after discharge & 184 & 27 & 35 \\
$\quad$ Total normal/abnormal & 206 & 25 & 211 \\
ANNPs & 17 & 1 & 231 \\
$\quad$ Referred from neonatal check & 223 & 26 & 18 \\
$\quad$ Referred after discharge & & 249 \\
Total normal/abnormal & & & \\
\hline
\end{tabular}

SHO, Senior house officer; ANNP, advanced neonatal nurse practitioner.

either directly from the neonatal check, from general practitioners, or through orthoptists.

All infants referred from these two hospitals with cardiac murmurs are assessed by a specialist registrar or consultant paediatrician, and, if a structural abnormality is suspected, a referral is made to the regional paediatric cardiology service. Similarly children referred by their general practitioner or community paediatrician with a cardiac murmur will initially be assessed by a consultant paediatrician, and referred on to the cardiologists if appropriate as above.

The paediatric cardiologists obtain a definitive diagnosis of structural cardiac abnormality by a combination of clinical examination and echocardiography. It is assumed that the vast majority of significant congenital cardiac abnormalities will present in infancy; our approach is consistent with previous studies. ${ }^{2}$ Infants presenting with acquired cardiac conditions (such as Kawasaki's disease) were excluded from the study.

Patients who fail to attend are offered two re-appointments, and then discharged. Hull is a stable population relatively isolated from other population centres and hospitals, and thus we feel confident that those abnormalities picked up subsequent to the neonatal period will still eventually be referred in to the specialist units listed above, rather than being "lost" to other centres.

Appropriate statistical analysis was performed using the two sample test for proportions. ${ }^{14}$

\section{Results}

A total of 527 infants were seen in the respective clinics and recruited into the study.

DIFFERENCES BETWEEN THE STUDY HOSPITALS The populations at each hospital did display some differences. In particular, Hull Maternity Hospital has a neonatal intensive care unit and accepts the high risk deliveries. Indeed, the overall rate of caesarian section was significantly higher at this hospital during the study

Table 2 Summary of sources and findings of ophthalmology referrals

\begin{tabular}{llll}
\hline & Normal eye & Abnormal eye & Total referred \\
\hline Paediatric SHOs & & 3 & \\
$\quad$ Referred from neonatal check & 4 & 6 & 7 \\
$\quad$ Referred after discharge & 2 & 9 & 15 \\
$\quad$ Total normal/abnormal & 6 & 6 & 10 \\
ANNPs & 4 & 0 & 5 \\
Referred from neonatal check & 5 & 6 & 15 \\
$\quad$ Referred after discharge & 9 & & \\
Total normal/abnormal & & & \\
\hline
\end{tabular}

SHO, Senior house officer; ANNP, advanced neonatal nurse practitioner.
(20.8\% of live births $v 18.5 \%$ at Castle Hill; $\mathrm{p}<0.05)$. However, importantly for the validity of this study, there was no difference between the two hospitals in the underlying rate of hip $(1.1 \%)$, eye $(0.3 \%)$, and cardiac $(0.6 \%)$ abnormalities presenting in infancy.

HIP

A total of 467 infants were referred to the specialist orthopaedic hip clinic at Hull Royal Infirmary during the course of the trial. Of these, 211 had been born at Hull Maternity Hospital, and 249 at Castle Hill hospital (table 1). Seven had been born elsewhere.

Hips were defined as abnormal either if they were dysplastic requiring either Pavlik harness or operative intervention or they showed Graf grade IIa changes on ultrasound. These grade IIa hips are borderline and felt worthy of follow up, as a proportion require subsequent intervention. In our study, 36 hips were classed initially as grade IIa, of which 18 normalised; two progressed to dysplasia requiring intervention, and 16 maintained their grade throughout the course of the study.

Paediatric trainees at Hull Maternity Hospital showed a sensitivity of $20 / 27(74 \%)$, with a positive predictive value of $20 / 176(11 \%)$. For infants referred on the basis of an abnormal clinical examination (rather than because of risk factors) the positive predictive value was 7/35 (20\%). ANNPs at Castle Hill Hospital referred with a sensitivity of $25 / 26(96 \%)$ and a positive predictive value of $25 / 231$ (11\%). Abnormal clinical examination showed a positive predictive value of $16 / 88(18 \%)$. The difference in sensitivity between SHOs and ANNPs was significant $(p<0.05)$, with no difference in positive predictive values ( $\mathrm{p} 0.5)$.

When six hour discharges were compared with standard neonatal checks across both hospital sites, there was no significant difference in effectiveness; however, only 21 babies referred to the hip clinic had had a six hour discharge.

Across all the infants from both hospitals, it was also possible to assess how helpful a particular clinical feature is in predicting an abnormal hip. In total, 217 babies were referred with breech presentation as the sole indication, of which 14 had abnormalities (positive predictive value 6\%). By comparison, 9/113 babies presenting solely with clicky hips (positive predictive value $8 \%$ ), 6/58 presenting solely with a positive family history of dysplastic hips (positive predictive value 10\%), and $22 / 30$ babies who were felt to have dislocated or dislocatable hips on the neonatal check (positive predictive value $73 \%$ ) were shown to have abnormal hips by the orthopaedic surgeon.

EYE

Thirty one infants were referred to ophthalmologists. Of these, 15 had been born at Hull Maternity Hospital and had had a neonatal check performed by trainee paediatricians; 15 had had their check performed by ANNPs at Castle Hill, and one child had been born elsewhere (table 2). 
The main reasons for referral were reduced/ absent red reflex (seven), family history of cataract (five), and maternal concern (four).

Infants referred after discharge were generally referred by the general practitioner (seven), consultant paediatrician (four), or orthoptist (two).

Ophthalmologist's findings that were classed as abnormal were: cataract; congenital ptosis; congenital Horner's syndrome; rubeosis iridis and retinal dystrophy; micro-ophthalmia with coloboma; total retinal detachment; septooptic dysplasia; hypoplastic optic nerves; and family history of congenital glaucoma and retinoblastoma as it was felt that follow up was important.

Paediatric SHO's displayed a sensitivity of $3 / 9$ or $33 \%$, with a positive predictive value of $3 / 7$ or $42 \%$. ANNPs in contrast showed a sensitivity of $6 / 6$ or $100 \%$, with a positive predictive value of $6 / 10$ or $60 \%$. The difference in sensitivity between SHOs and ANNPs was significant $(p<0.05)$. The difference in positive predictive values was not significant $(\mathrm{p}<0.1)$.

Those referred from either hospital with reduced/absent red reflex (seven) were mostly abnormal, with five cataracts and one retinal detachment being identified by the ophthalmologists. Family history of disease was a less fruitful marker for disease, with eight of 11 being completely normal, one (history of retinoblastoma) needing further expert opinion, and two were currently normal but requiring long term review.

CARDIAC

Thirty nine infants were referred to the paediatric cardiologists. Of these, 28 were referred because of the presence of a murmur which was felt not to be innocent, and 11 for other reasons such as cyanosis or dysmorphism.

Twenty five of the infants referred for cardiology opinion had been born at Hull Maternity Hospital, 12 at Castle Hill Hospital, and two elsewhere (table 3 ).

Abnormalities included: isolated ventricular septal defect (10); transposition of the great arteries (five); isolated atrial septal defect (three); Tetralogy of Fallot (two); pulmonary stenosis (four); and coarctation (one).

Of the five infants who presented with transposition of the great arteries, only one presented before discharge from hospital, with profound cyanosis in the first 24 hours of life. Three infants presented at between 6 and 14 days of age, with cyanosis being noted either by

Table 3 Summary of source and findings of cardiac referrals

\begin{tabular}{llll}
\hline & Normal heart & Abnormal heart & Total referred \\
\hline Paediatric SHOs & 5 & 7 & \\
$\quad$ Referred from neonatal check & 5 & 11 & 12 \\
$\quad$ Referred after discharge & 2 & 18 & 13 \\
$\quad$ Total normal/abnormal & 7 & 5 & 25 \\
ANNPs & 1 & 5 & 6 \\
$\quad$ Referred from neonatal check & 1 & 10 & 6 \\
$\quad$ Referred after discharge & 2 & 12 \\
$\quad$ Total normal/abnormal & & & \\
\hline
\end{tabular}

SHO, Senior house officer; ANNP, advanced neonatal nurse practitioner. the community midwife or general practitioner. One infant presented at five months of age through the community paediatricians with a murmur.

Only $5 / 10(50 \%)$ of isolated ventricular septal defects presented on the routine neonatal check, with the rest being detected subsequently.

Only two referrals were made from a six hour discharge check, but interestingly both were abnormal, one infant having Tetralogy of Fallot and one having pulmonary stenosis.

Paediatric SHOs displayed a sensitivity of $7 / 18(39 \%)$, with a positive predictive value of $7 / 12(58 \%)$. ANNPs had a sensitivity of $5 / 10$ $(50 \%)$, with a positive predictive value of $5 / 6$ $(83 \%)$. The differences between SHO and ANNP for both sensitivity and positive predictive value were not significant $(p \sim 0.1)$.

\section{INFANTS FAILING TO ATTEND THE SPECIALIST} CLINICS

The rate of failure to attend during the study was $10 \%$. There was no reason to suspect that this would cause any bias between the two groups. However, to validate further the findings in the largest arm of the study, additional surveillance of the hip clinic was performed between 1 April 2000 and 31 March 2001 for any children presenting later than 1 year of age with developmental dysplasia of the hip. No such children presented.

\section{Discussion}

In two arms of the study, the ANNPs were significantly better at detecting abnormalities during the neonatal check, with the underlying trend being similar in the smaller third cardiac arm, though not significant. Positive predictive values were the same between the two groups in each arm of the study, with an evident trend towards ANNPs having a better positive predictive value with cardiac and eye abnormalities.

These findings have two major implications. Firstly, it shows that ANNPs are effective in performing the neonatal examination. Taken with other studies showing that ANNPs are as effective as doctors at newborn resuscitation, ${ }^{15}$ and are more cost effective than doctors when providing neonatal intensive care under the supervision of a neonatologist, ${ }^{16}$ this provides a sound evidence base for their practice. Secondly, it raises points about the different models of education that have been traditionally used in medical and nursing training. ANNP training in neonatal examination is much more structured and the evaluation is competency based. Many paediatric SHOs are either expected to ensure their own competency by reading and other forms of self directed learning, ${ }^{8}$ or are given a short demonstration of the neonatal check and a request to have any abnormalities checked out by a senior colleague. Previous studies have shown that only $37 \%$ of maternity units train their junior paediatric staff using a "Baby Hippy" hips simulator. ${ }^{12}$ This study appears to suggest that the 
nursing model may be more efficacious when training personnel to perform the neonatal check.

There were differences between the populations attending the two study hospitals, with high risk deliveries being booked at Hull Maternity Hospital. Importantly, we showed that there was no difference between the two hospitals in the underlying abnormality rate.

The failure to attend rate $(10 \%)$ at the specialist clinics may have introduced a degree of bias into the study. However, the parents of these babies were offered two re-appointments, and still did not present. It is assumed that most of these babies were in fact normal; this is supported by the fact that none of this birth cohort presented between the ages of 1 and 2 with abnormal hips. The positive predictive value of both groups may have been slightly improved, however.

At both hospitals, hip abnormalities were generally referred directly without middle grade or senior review. Suspected abnormalities of the eyes or heart detected by an SHO or ANNP were reviewed by the middle grade or consultant before referral, which may have improved the positive predictive values of both groups', but did not affect their sensitivities.

Current practice for diagnosing developmental dysplasia of the hip relies on Graf's sonographic technique. ${ }^{13}$ Previous large studies have suggested that grade IIa (borderline) hips mature spontaneously in $89 \%$ of cases, and $11 \%$ need abduction. ${ }^{17}$ Our study showed results consistent with this, with $6 \%$ requiring abduction, $50 \%$ regressing, and $44 \%$ still requiring further observation in the orthopaedic clinic. The incidence of dysplastic hips is found to be about $1.1 \%{ }^{17}$ with universal neonatal ultrasound screening; in our study across two hospitals with a combined rate of 5027 live births, we found an identical incidence of $1.1 \%$, which further validates our study. We found that $6 \%$ of babies born in the breech position had unstable or borderline hips; this is also identical with a previous larger study. ${ }^{18}$

In both groups, the proportion of babies referred who had abnormal hips was low (positive predictive value $11 \%$ ). This is in large part due to the large volume of babies referred because of either breech presentation or family history as the sole indication, but is still a 10 -fold increase in risk of abnormality when compared with the background population in this study.

The most significant marker for developmental dysplasia of the hip on the neonatal check is a dislocated or dislocatable hip (this is distinct from "clicky" hips, which were analysed separately). Almost three quarters (73\%) of babies with this clinical finding were shown to have abnormal hips. Taking all the babies in the study who were shown to have abnormal hips, $34 \%$ were referred with a dislocated or dislocatable hip as the sole reason for referral, with no other risk factors being present. This shows the vital importance of proper training and execution of the clinical examination of hips during the neonatal examination, as otherwise these babies would present late.
Previous studies of the detection of congenital cataract have shown that up to $33 \%$ do not present until after 1 year of age. ${ }^{4}$ By definition, this study purely assessed infants presenting to the ophthalmologists up to 1 year of age, which clearly introduces the possibility of bias into our results. However, there is no reason to suspect that this bias should be distributed unequally between the two groups. What was of interest was the very high pick up rate for serious abnormalities (86\%) when the red reflex is found to be absent. This further emphasises the importance of this clinical sign at the neonatal check, especially in view of the need for early surgery before 6 weeks of age in children with cataract or retinal detachment to optimise outcome. ${ }^{5}$

Our study showed that the neonatal check failed to detect $57 \%$ of babies with congenital heart disease; in fact, only $33 \%$ of such infants presented because of the detection of a murmur at the neonatal check. Although numbers in this study with congenital heart disease were small, it is encouraging that, in this respect, our findings concur with much bigger studies looking at this subgroup of babies. ${ }^{219}$ Of our infants referred to the paediatric cardiologists because of the presence of a murmur, $68 \%$ had a structural abnormality of the heart. This is a higher proportion than the $54 \%$ shown in previous studies, ${ }^{19}$ probably because of our practice of paediatric consultant review of all murmurs before referral to the paediatric cardiologists.

This study has shown that ANNPs are more effective than paediatric SHOs in detecting abnormalities during the neonatal check. This has significant implications in the current climate of NHS workforce planning reviews, ${ }^{2021}$ and also challenges the methods of training given to paediatric SHOs to perform this task.

We would like to thank Dr J L Gibbs of the Yorkshire Heart Centre for access to the cardiology patient database.

1 Hall DMB. The role of the routine neonatal examination [editorial]. BMF 1999;318:619-20.

2 Wren C, Richmond S, Donaldson L. Presentation of congenital heart disease in infancy: implications for routine examination. Arch Dis Child Fetal Neonatal Ed 1999;80:F49-53.

3 Meberg A, Otterstad JE, Froland G, et al. Early clinical screening of neonates for congenital heart defects: the cases we miss. Cardiology in the Young 1999;9:169-74.

4 Rahi JS, Dezateux C. National cross sectional study of detection of congenital and infantile cataract in the United Kingdom: role of childhood screening and surveillance. The British Congenital Cataract Interest Group. BMF 1999;318:362-5.

5 Birch EE, Stager DR. The critical period for surgical treatment of dense congenital unilateral cataract. Invest Ophthalmol Vis Sci 1996;37:1532-8.

6 Glazener CMA, Ramsey CR, Campbell MK, et al. Neonatal examination and screening trial (NEST): a randomised, controlled, switchback trial of alternative policies for low risk infants. BMf 1999;318:627-32.

7 Jones D. Neonatal detection of developmental dysplasia of the hip (DDH) [editorial]. F Bone foint Surg $[\mathrm{Br}]$ 1998;80:943-5

8 Walker D. Role of routine neonatal examination. It probably makes more sense for other staff to carry out neonatal examinations [letter]. BMF 1999;318:1766.

9 Doherty LE. The advanced neonatal nurse practitionerroom for one more? Fournal of Neonatal Nursing 1996; 2(4): 23-7.

10 Allen F. Advanced neonatal practice: The northern experience so far. Fournal of Neonatal Nursing 1997;3:6-8.

11 Dillon A, George S. Advanced neonatal nurse practitioners in the United Kingdom: where are they and what do they do? F Adv Nurs 1997;25:257-64. 
12 Dezateux C, Godward S. A national survey of screening for congenital dislocation of the hip. Arch Dis Child $1996 ; 74: 445-8$.

13 Graf R. The diagnosis of congenital hip-joint dislocation by the ultrasonic compound treatment. Arch Orthop Trauma Surg 1980;97:117-33.

14 Colton T. Statistics in medicine. Boston: Little, Brown, and Company, 1974

15 Britton JR. Neonatal nurse practitioner and physician use on a newborn resuscitation team in a community hospital. f Pediatr Health Care 1997;11:61-5.

16 Bissinger RL, Allred CA, Arford PH, et al. A costeffectiveness analysis of neonatal nurse practitioners. Nursing Economics 1997;15:92-9.
17 Falliner A, Hahne HJ, Hassenpflug J. Sonographic hip screening and early management of developmental dysplascreening and early management of developmental
sia of the hip. F Paediatr Orthop B 1999;8:112-17.

18 Holen KJ, Tegnander A, Terjesen T, et al. Ultrasonographic evaluation of breech presentation as a risk factor for hip dysplasia. Acta Paediatr 1996;85:225-9.

19 Ainsworth SB, Wyllie JP, Wren C. Prevalence and clinical significance of cardiac murmurs in neonates. Arch Dis Child Fetal Neonatal Ed 1999;80:F43-5.

20 Department of Health. A health service for all the talents. http://www.doh.gov.uk/pdfs/workforce.pdf.

21 Department of Health. The NHS plan. http:// www.doh.gov.uk/nhsplan/nhsplan.htm.

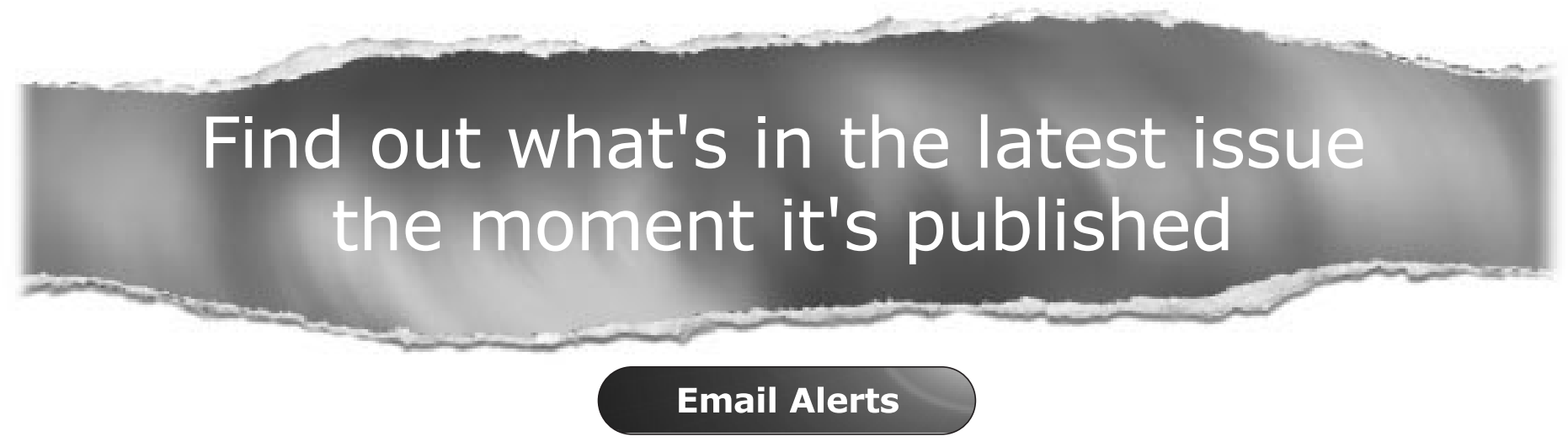

Sign up to receive the table of contents by email every month. You can select from three alerts: Table of Contents (full), TOC Awareness (notice only); Archives of Disease in Childhood related announcements.

www.archdischild.com 